\title{
A new decoding and transcription device (DTD) for SSR keyboard data
}

\author{
MARK MORRIS and EVELYN SHAW \\ Department of Biological Sciences, Stanford University, Stanford, California 94305
}

\begin{abstract}
This report describes a device for decoding and transcribing behavioral observations encoded on magnetic tape with the SSR (Stephenson \& Roberts, 1977) keyboard. The decoding and transcription device (DTD) uses two microprocessors to reduce the data and transcribe them into a computer-compatible medium, or directly into a computing system. The SSR system and its uses are briefly described, as is a particular implementation of the system incorporating the DTD for transcription over a phone line into a timesharing computer.
\end{abstract}

The advent of the computer has inspired major changes in the techniques of data collecting, processing, and analyzing among scientists studying animal and human behavior. That more scientists are not employing the new systems may be (1) a reflection of the fear of not fully understanding the steps, (2) a concern about the intricacies of constructing such systems in individual laboratories, or (3) the result of high costs. Such deterrents are gradually being demolished. This paper describes a system that requires only the integration of existing devices. The system is based on the use of a keyboard for encoding observations in a form amenable to automated processing.

\section{HISTORICAL SUMMARY}

Keyboards that connect to multichannel strip-chart recorders (e.g., the Esterline-Angus) have been in use for many years. While representing an advance over data-taking with notebook and stopwatch, they have a number of shortcomings. The recorders are bulky and unsuitable for field work, and the data-storage medium, paper strip charts, is also rather cumbersome. Their most negative feature, however, is the vast amount of time required for transcription into a computercomprehensible format.

More sophisticated systems have been devised for direct transcription into a computer medium. Other units, particularly those designed for use in the field, have used intermediate storage on magnetic tape, with transcription as a separate step.

One approach to direct transcription in the laboratory involves encoding keystrokes and the time of their occurrence on punched paper tape (Fernald \& Heinecke, 1974; Tobach, Scheirla, Aronson, \& Laupheimer, 1962). While solving the transcription

This work was supported in part by Grant OCE76-08546 to E. Shaw from the National Science Foundation. Dr. Shaw is a recipient of a Research Scientist Award MH 05349 from the National Institutes of Health. Detailed information on circuitry and microprocessors may be obtained by writing the authors. problem, this technique has the other disadvantages of strip-chart recording. Moreover, many computing facilities no longer maintain paper-tape readers.

Beauchamp and Scobie (1973) describe a high-speed, 120-channel event recorder that transcribes onto nine-track digital magnetic tape. This type of system is powerful and versatile, but it is restricted to use in the laboratory.

Systems that encode the occurrence of individual keystrokes store this information with maximum compactness. This efficiency makes it possible for the commercially available Datamyte (Torgerson, 1977) to use a semiconductor memory for data storage. But there are limitations to this encoding scheme, as well.

The Datamyte has a practical limit of 5 keypresses/ sec; faster keying may result in loss of data. The systems of Dawkins (1971) and Gass (1977) are similarly constrained. Furthermore, the Dawkins' system and the Datamyte are restricted to a rather small character set. While these devices are adequate for some keyboard applications, there is no room in the encoding procedure for recording any other sort of digital data. Moreover, with a semiconductor memory, there is no capacity for recording a simultaneous voice-note record. The memory contents are vulnerable to power supply failure, and when the memory is full, it must be transcribed before more data can be collected.

The DCR-II event recorder (Celhoffer, Boukydis, Minde, \& Muir, 1977) escapes some of these disadvantages. This device can accommodate 64 event channels, and records up to 100 events/sec on digital cassette tape. Direct computer access is necessary for transcription, however.

WRATS, developed by White (1971), and an early version of the SSR keyboard (Stephenson, Smith, \& Roberts, 1975) both employ phase encoding to store the state of all the keys on the keyboard several times per second on magnetic tape. This is a relatively "inefficient" way to store keyboard data, because keyboard images. or sweeps, must be recorded even when no keys are being pressed. Nevertheless, this redundancy has 
advantages for protecting the data. Two or more keys pressed simultaneously are simply so recorded. A tape flaw may make a particular sweep indecipherable, but any change that occurred can still be found in the next sweep. Also, these systems accommodate the recording of other kinds of digitally formatted data. Voice notes can be recorded simultaneously on multitrack tape.

When digital keyboard data are stored on audio magnetic tape, complex procedures are required to decode the data before transcription into a computer. Both WRATS and the SSR system have required direct access to a minicomputer for decoding and transcription. The advent of the microprocessor, however, has made it possible to separate decoding and the first steps in data reduction from the minicomputer. This has the advantage of saving machine time for other operations, even if direct access to a minicomputer is available. Our system employs two microprocessors for decoding and transcription. The current version of the Datamyte uses a microprocessor in each keyboard to control both recording and transcription. This makes each Datamyte keyboard a self-contained data collecting system, at the price of redundancy and unnecessary expense for a project involving many keyboards.

Solid state memory technology has advanced to the point where it is practical to record thousands of individual keystrokes on single-chip memory modules or digital cassette tape with a portable battery-operated device. In several years, it will be practical to so record hundreds of thousands of keyboard images. Until then, a tradeoff exists between the simplicity of recording keystrokes in a digital medium and the security and versatility of recording keyboard images on tape.

\section{THE SSR SYSTEM}

The SSR keyboard provides a very generalized and versatile method for recording behavioral observations. The translating device developed for our laboratory has similar advantages for the process of transcribing SSR keyboard data into a computer or computercompatible medium.

Keyboard versatility is based on a number of features that permit many kinds of observations to be made in the laboratory. These observations can be stored in a compact and convenient manner. Since the keyboard is light and battery operated, with a battery-operated tape recorder, it can be used in the field as well. The keyboard and its uses are described in detail by Stephenson and Roberts (1977).

With the SSR system, data are stored on magnetic tape, a compact and readily available medium. By using a stereo tape recorder, the data are recorded on one track and, simultaneously, the observer's spoken comments are recorded on the other. The keyboard provides 48 keys. These include 26 alphabetic characters, 10 numerals, and 12 other symbols. The state of the keyboard is encoded on the tape
20 times/sec. This provides greater precision in measuring the experimenter's operation of the keyboard than the experimenter is likely to be able to bring to bear on his observations.

The keyboard may be used as a 48-channel event recorder by assigning one key to each behavior. If keys are used in combinations, any number of behaviors may be coded, each represented by a unique sequence of keystrokes. It may even be used to record observations in a text format, as if typed on a typewriter.

Other types of data (indeed, any information that can be converted to a digital format) may be encoded on tape through the keyboard. For example, external switches may be substituted for specific keys. Thus, a pressure switch might be used to code for the presence of an animal in a particular location. A continuously varying electrical signal, such as the voltage produced by a thermocouple or from a "joystick," that indicates an animal's position can be digitized and recorded through the keyboard, either in place of some keys or on alternate keyboard images.

After the behavioral observations are recorded, they are then entered into a computer for analysis. The SSR group developed a set of electronic circuits and computer programs for transcribing SSR data tapes, but there are some difficulties with their transcription system. The user must build and "debug" the circuitry himself, and the design requires direct access to a minicomputer. These two factors may constitute a major obstacle to "starting up" with the SSR system for a researcher without ready access to these facilities. To make our SSR system work, a decoding and transcribing device (DTD) for SSR data tapes was developed. Although based on the SSR design, our system has much broader applicability. In conjunction with a terminal and other standard computer peripherals, it can be used to transcribe data into computer-interpretable media, such as digital magnetic tape or punched cards. Or it may be used for direct transcription into essentially any sort of interactive computing facility, from a dedicated minicomputer to a large timesharing system.

\section{DATA TRANSCRIPTION TO A TIMESHARING SYSTEM}

In our laboratory, the SSR system is used for study of piscine reproductive strategies. The taped data are decoded, translated into a more compact form, then transmitted over a phone line to Stanford's computing facility (Figure 1). The following description is intended to provide some insight into the theory of operation of the SSR keyboard and our decoding and transcription device, with a minimum of technical detail. This is only one of a number of ways whereby the data tapes might be transcribed into a computer, using the DTD.

First, data are typed out, in a code of the user's choice, on the SSR keyboard. The string of characters 


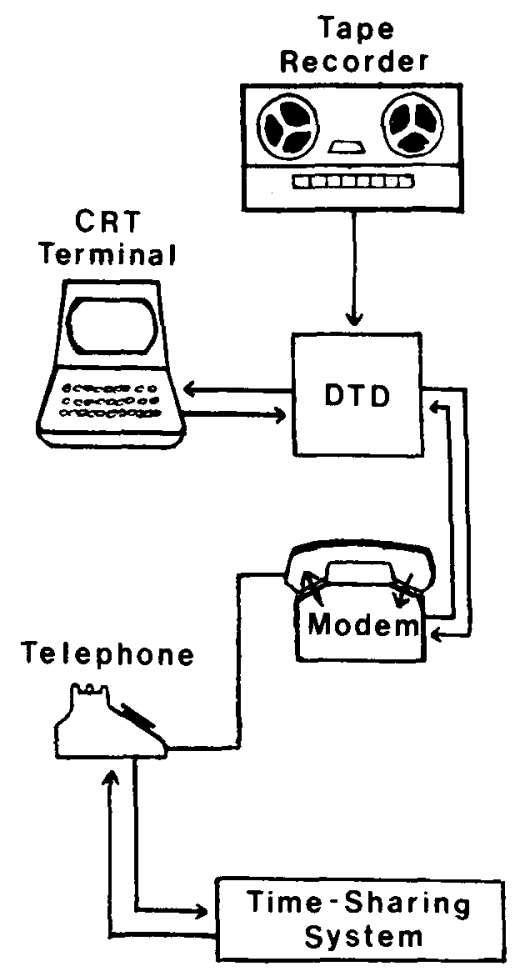

Figure 1. Data are decoded from the tape-recorder output by the DTD. They are then sent through the modem and telephone by a timesharing system. The timesharing system echoes the data back to the DTD, where they are checked for errors and then sent to the terminal for display.

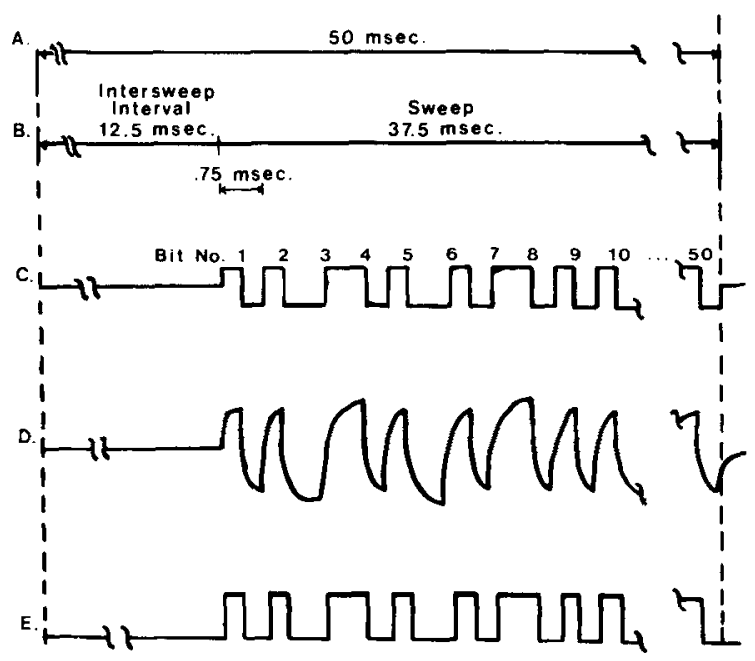

Figure 2. (A) A keyboard image, or sweep, is recorded every 50 msec. (B) Each 50 msec contains a 12.5 -msec silent period; the remaining $37.5 \mathrm{msec}$ is divided into 50.75 -msec blocks, within which the state of the keyboard is encoded. (C) The signal sent to the tape consists of a series of rectangular pulses. (D) When the tape is played back, the signal is no longer so well defined. (E) The signal-conditioning circuit converts this back into a form similar, but not identical, to the original signal; the signal is at a low, rather than an intermediate value during the intersweep interval, and there is some error in the location of transitions.
"A13" might, for example, represent the initiation of Behavior A by Animal 1 toward Animal 3. The time that the behavior is initiated is marked by the time that the "t" key is pushed.

The keys are push-on/push-off types. The state of all the keys on the board is examined 20 times $/ \mathrm{sec}$, and a "keyboard image" is produced (Figure 2). Thus, the time at which a key was pushed is known to within $.05 \mathrm{sec}$. The order of keypresses is preserved if they are separated by more than $.05 \mathrm{sec}$.

The keyboard images are then fed into the microphone input of a tape recorder and preserved as "sweeps" on the tape. The fashion in which each keyboard image is stored on tape is simple, in principle. The output to the tape recorder during each $.05 \mathrm{sec}$ $(50 \mathrm{msec})$ is divided into two parts: a 12.5 -msec silent period and a 37.5 -msec sweep period. The sweep period is divided into 50.75 -msec blocks. The state of each key, whether on or off, is "phase encoded" in 1 of these 50 blocks. That is, the output to the tape is high in the first half and low in the second half of the block if the key is on, or low and then high if the key is off. The first and last blocks are reserved for marking the beginning and end of the sweep period. The keys representing the 26 alphabetic characters, the 10 decimal numerals, and 12 other symbols are encoded in the remaining 48 blocks.

In the next stage of the transcription process, the tape is played back; the preamplifier output is directed into a signal-conditioning circuit, which converts the signal on the tape into a signal compatible with digital logic circuitry (see Figure 2).

Next, the signal is examined by a microprocessor. This microprocessor, Micro-S, determines whether sweeps are present. When a sweep is detected, Micro-S determines whether each of the keys is on or off and stores this information as a series of ones and zeros. The sampling process by which the state of the keys is determined can be modified by the user from the CRT terminal for tape playback speeds deviating from the normal values of one, two, or four times the record speed.

The 48 ones and zeros, or bits, representing one sweep are sent to a second microprocessor. The second microprocessor, Micro-C, does several things with this information. It adds one to, or increments, the sweep count, a number representing the total number of sweeps that have been received. It then determines which keys, if any, have changed state. Micro-C compares the last three sweeps received. It compares the previous sweep with the sweep before that, and with the sweep just received. If the comparisons show that a bit has changed on the previous sweep and has not changed back on the sweep just received, the change is interpreted as being due to a legitimate keystroke. If, however, a bit changes and then changes back immediately, the change is interpreted as a tape flaw and disregarded (see Figure 3). 


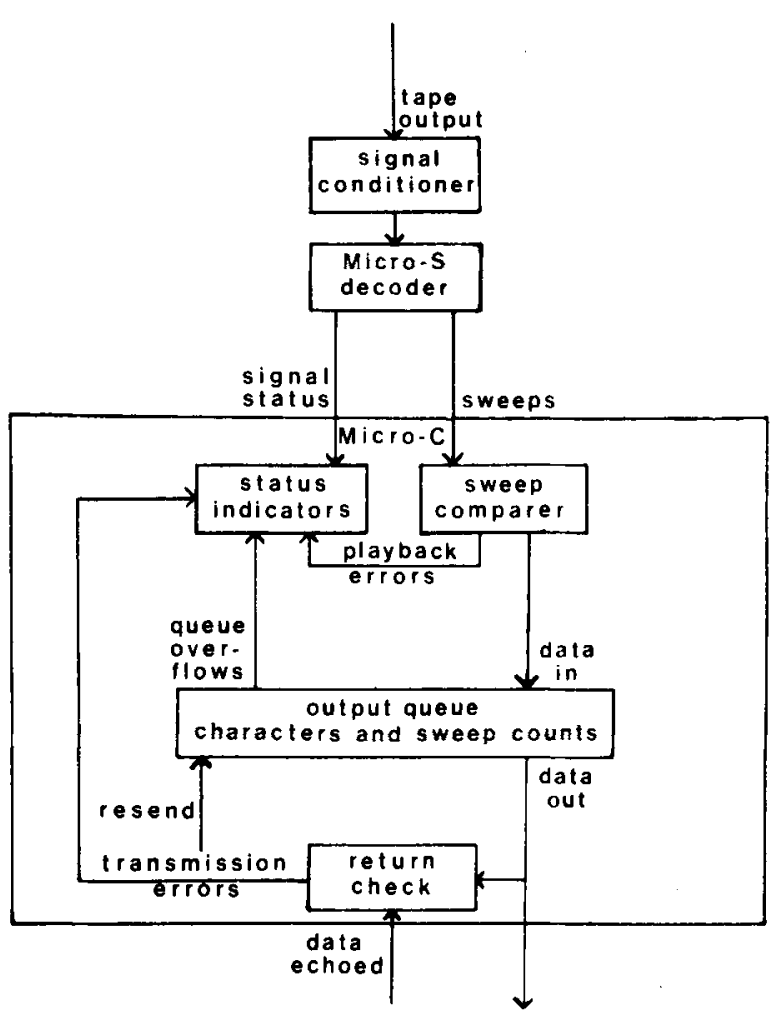

Figure 3. Outline of DTD operations during transcription. The signal from the tape recorder is conditioned and then decoded by Micro-S. Micro-S notifies Micro-C of each sweep, and of silent periods lasting more than $.25 \mathrm{sec}$. Each decoded sweep is passed to Micro-C. Valid keystrokes, as well as tape flaws in each sweep, are detected by comparisons with the previous and subsequent sweeps. Each keystroke and the time of its occurrence is loaded into an output queue. The data are sent out and, as they are echoed by the receiving system, checked against what was sent. When transmission errors are detected, the data are sent again. Status lights and CRT terminal messages inform the user of transcription status and errors.

The volume of the data that must be sent is reduced by noting only the times when a key changes, from the stream of information on whether it is up or down. Since most of the time, most of the keys are not changing, a major reduction can be achieved in this way, with no loss of information.

It is not practical to send raw data from Micro-S over the phone line, simply because the raw data are generated faster than they can possibly be transmitted over a 300-baud modem. Raw data are generated at $48 \mathrm{bits} / \mathrm{sweep} \times 20$ sweeps $/ \mathrm{sec} \times 1$ character $/ 6$ bits $=$ 160 characters/sec, if the tape is played back at the speed at which it was recorded. With the most commonly used modems, however, transmission is limited to 30 characters/sec or less. If keys are pushed at an average rate of $1 / \mathrm{sec}$, then the data reduction scheme outlined above requires that only 4 characters/sec be sent. Playback can be accelerated two or four times without approaching the 30-characters/sec limit.

Each character and associated sweep count is stored as 4 bytes in a first-in/first-out output queue. When a legitimate keystroke is detected in the raw data from the sweeps, Micro-C "looks up" in a table the ASCII code word that corresponds to that key. The ASCII code is a widely used code for representing letters, numbers, and a variety of other symbols in the form of binary numbers. All of the common keyboard symbols, such as A, 3, and ?, are represented, each by its own byte, or 8-digit binary number. Other codes, such as IBM's EBCDIC, can easily be substituted.

The contents of the output queue are then sent to an Omnitech modem (Omnitech Corporation), a standard device for sending and receiving digital information through a telephone. The modem translates the electric signals that it receives into audio signals and sends them over a telephone line to the large computer in a timesharing system. The program in the main computer that receives and stores the data echoes the characters and sweep counts. These come back to the microprocessor through the modem, and are then compared with what was sent. When an error is detected, queue pointers are moved back, so that the data are sent again, and the receiving program splices out the error.

Commands from an ADM-3A (Lear Siegler, Inc.) CRT computer terminal initiate and end the process of data transcription. Indicator lights as well as messages to the terminal inform the user of system status. The CRT terminal is also useful for typing in and using other programs that further reduce and analyze the data.

\section{CONCLUSION}

This combination of the SSR system with the DTD results in several significant advantages, the most important of which is its convenience for the user. The hardware involved in the transcription process requires a minimum of adjustment and attention. Data can be transcribed, with automatic transmission-error correction, from wherever a telephone and wall socket can be reached. Any amount of data may be collected before transcription is required. The transcription operation can readily be shifted from one computing facility to another, in most cases. The component circuit boards of the DTD can be incorporated directly into a laboratory microcomputer.

The SSR keyboard is particularly suitable for recording complex observations, in the field or laboratory, along with voice notes or digitized measurements. In spite of the cost of the DTD, the combined SSR and DTD system is among the most economical of such systems for projects involving several keyboards. Keyboards are available from Semeiotic Systems Corporation, 13 Homestead Court, Madison, Wisconsin 53711. The basic keyboard, as of August 1977, lists for $\$ 650$. The interactive FORTRAN version of the DTD is available from Carry Bit Corporation, 945 Van Auken Circle, Palo Alto, California 94303, for $\$ 4,500$. 


\section{REFERENCES}

Beauchamp, J., \& Scobie, S. R. A high-speed computercompatible event recorder. Behavior Research Methods \& Instrumentation, 1973, 5, 418-424.

Celhoffer, L., Boukydis, C., Minde, K., \& Muir, E. The DCR-II event recorder: A portable high-speed digital cassette system with direct computer access. Behavior Research Methods \& Instrumentation, 1977, 9, 442-446.

DAwkins, R. A. A cheap method for recording behavioral events for direct computer access. Behaviour, 1971, 40, $162 \cdot 173$

Fernald, R. D., \& Heinecke, P. A computer-compatible multipurpose event recorder. Behaviour, 1974, 48, 268-275.

Gass, C. L. A digital encoder for field recording of behavioral, temporal, and spacial information in a directly computer. accessible form. Behavior Research Methods \& Instrumentation, 1977, 9, 5-11.

Stephenson, G. R., \& Roberts, T. W. The SSR System
7: A general encoding system with computerized transcription. Behavior Research Methods \& Instrumentation, 1977. 9. 434.491.

Stephenson, G. R., Smith, D. B. P., \& Roberts, T. W. The SSR system: An open format event recording system with computerized transcription. Behavior Research Methods \& Instrumentation, 1975, 8, 256-277.

TOBach, E., SChneirla, T. C., ARonson, L. R., \& LAUPhEIMER, R. The ATSL: An observer to computer system for a multivariate approach to behavioral study. Nature, 1962, 194, 256-258.

Torgerson, L. Datamyte 900. Behavior Research Methods \& Instrumentation, 1977, 9, 405-406.

WHTE, R. E. C. Wrats: A computer-compatible system for automatically recording and transcribing behavioral data. Behaviour, 1971, 40, 135-161.

(Received for publication January 9, 1978; revision accepted June 20, 1978.) 